\title{
A method of accurate guiding for LAMOST $\dagger$
}

\author{
Y. Yu ${ }^{1}$, Z. X. Qi ${ }^{1,2}$, Y. D. Mao ${ }^{1}$, Z. H. Tang ${ }^{1}$ and M. Zhao ${ }^{1}$ \\ ${ }^{1}$ Shanghai Astronomical Observatory, Chinese Academy of Sciences, Shanghai 200030, China \\ email: yuy@shao.ac.cn \\ ${ }^{2}$ Graduate School of the Chinese Academy of Sciences, Beijing 100039, China
}

\begin{abstract}
The main optical instruments of the Large Sky Area Multi-Object Fiber Spectroscopic Telescope (LAMOST) are installed separately. Guiding is realized by adjusting the normal direction of the mirror MA. In order to keep the central star imaged on the rotation center of the fiber plug plate, the link between celestial and fiber plug plate coordinate systems has to be established. Some local parameters contain errors so that the normal direction of the MA calculated beforehand may not be accurate enough. The execution error of the telescope also drives the image of the central star to deviate from the rotation center. Therefore, in the process of observations the correction has to be calculated and applied periodically. Here we present the method of calculating the guiding parameters as well as the correction parameters for the LAMOST.
\end{abstract}

Keywords. astrometry, techniques: miscellaneous

\section{Principle of calculating the guiding parameter}

LAMOST is a special reflecting Schmidt telescope (Wang et al. 1996). Its focal length is $20 \mathrm{~m}$ and the field of view is 5 degrees. 4000 optical fibers are going to be installed in a fiber plug plate laid on the focal plane of the telescope to perform multi-object fiber spectroscopic observations A total of 4 CCDs are installed in the plate to perform the guiding. Different from general purpose telescopes, the Schmidt correction mirror MA, main mirror MB and fiber plug plate are all fixed with respect to ground. Guiding is realized by adjusting the normal direction of the MA. This determines the novelty of realizing the guiding. During observations the central star is required to be continuously imaged onto the rotation center of the fiber plug plate. So the normal direction of the $\mathrm{MA}$ is given by

$$
\vec{n}=<\vec{S}_{00}^{*}+\vec{S}_{00}>
$$

where $\vec{S}_{00}^{*}$ and $\vec{S}_{00}$ represent the direction of the central star and the rotating center of the fiber plug plate respectively. In order to perform calculation, both of them should be expressed in horizontal coordinate system, i.e.

$$
\begin{gathered}
\vec{S}_{00}^{*}=[\mathbf{N}]\left(\begin{array}{c}
\cos \alpha_{00} \cos \delta_{00} \\
\sin \alpha_{00} \cos \delta_{00} \\
\sin \delta_{00}
\end{array}\right)=[\mathbf{Z}] \bar{R}_{2}\left(90^{0}-\phi\right) \bar{R}_{3}\left(S_{l}\right)\left(\begin{array}{c}
\cos \alpha_{00} \cos \delta_{00} \\
\sin \alpha_{00} \cos \delta_{00} \\
\sin \delta_{00}
\end{array}\right), \\
\vec{S}_{00}=[\mathbf{F}]\left(\begin{array}{c}
0 \\
0 \\
1
\end{array}\right)=[\mathbf{Z}] \bar{R}_{3}\left(-A_{00}\right) \bar{R}_{2}\left(-90^{\circ}+H_{00}\right)\left(\begin{array}{c}
0 \\
0 \\
1
\end{array}\right),
\end{gathered}
$$

$\dagger$ Supported by the National Natural Science Foundation of China(Grant Nos. 10673026, 10333050, and 10573018) and Science \& Technology Commission of Shanghai Municipality(06DZ22101). 
where $[\mathbf{N}],[\mathbf{F}]$ and $[\mathbf{Z}]$ represent equatorial, fiber plug plate, and horizontal coordinate system; $A_{00}$ and $H_{00}$ are horizontal coordinates of the rotation axis of the fiber plug plate, and $\phi$ and $S_{l}$ are the astronomical latitude of the MA center and the local sidereal time.

On the other hand, the rotation angle of the fiber plug plate should be adjusted to compensate for the diurnal rotation of the image field and differential rotation caused by atmospheric refraction and aberration ( $\mathrm{Li}$ et al. 2006). Based on the reflection law, the direction vector of the $i$-th guide stars is calculated and expressed in the fiber plug plate plane coordinate system $[\mathbf{F}]$ by

$$
\vec{S}_{i}=<2\left(\vec{S}_{i}^{*^{\prime}} \vec{n}\right)-\vec{S}_{i}^{*}>=[\mathbf{F}]\left(\begin{array}{c}
\cos \theta_{i} \sin \rho_{i} \\
\sin \theta_{i} \sin \rho_{i} \\
\cos \rho_{i}
\end{array}\right) .
$$

Then equation (1.5) is adopted to determine the rotation angle, assuring that each guide star is located near the center of its corresponding CCD. Hereafter $\lambda i$ is the coordinate parameter of the $i$-th CCD center in the fiber plug plate.

$$
\sigma=\frac{\sum_{i=1}^{4}\left(\theta_{i}-\lambda_{i}\right)}{4}
$$

\section{Detecting deviations of the guiding parameters}

In the whole process of observation, correction has to be calculated and applied periodically by observing guide stars on surrounding CCDs.

The theoretical coordinates of the guide stars in the fiber plug plate system $[\mathbf{F}]$ can be calculated based on their catalog positions, i.e.

$$
\vec{S}_{i c}=<2\left(\vec{S}_{i}^{*^{\prime}} \vec{n}\right)-\vec{S}_{i}^{*}>=[\mathbf{F}]\left(\begin{array}{c}
\cos \lambda_{i c} \sin \gamma_{i c} \\
\sin \lambda_{i c} \sin \gamma_{i c} \\
\cos \gamma_{i c}
\end{array}\right)
$$

The corresponding observational coordinates can be obtained from CCD observations and expressed as

$$
\vec{S}_{i o}=[\mathbf{F}]\left(\begin{array}{c}
\cos \lambda_{i o} \sin \gamma_{i o} \\
\sin \lambda_{i o} \sin \gamma_{i o} \\
\cos \gamma_{i o}
\end{array}\right)
$$

Then the observation equation is

$$
\vec{S}_{i o}-\vec{S}_{i c}=\Delta \vec{S}_{00}+\Delta \sigma \sin \gamma_{i c}<\vec{S}_{00} \times \vec{S}_{i c}>.
$$

Using 4 guide stars, offset of the central star relative to the rotating center and rotation angle can be calculated together. Consequently, correction of the normal direction of the MA can be calculated by

$$
\Delta \vec{n}=-\frac{1}{\left|\vec{S}_{00}^{*}+\vec{S}_{00}\right|}\left(<\vec{S}_{00}^{*}+\vec{S}_{00}>\times\left(<\vec{S}_{00}^{*}+\vec{S}_{00}>\times \Delta \vec{S}_{00}\right)\right) .
$$

\section{References}

Li, J. L., Zhang, B., Yu, Y., Qi. Z. X. \& Zhao, M. 2006, Chin. J. Aston. Astrophys. 6, 495

Wang, S. G., Su, D. Q., Chu, Y. Q., Cui, X. Q. \& Wang, Y. N. 1996, Applied Optics 35, 5155 